University of Wollongong

Research Online

Faculty of Engineering and Information

Faculty of Engineering and Information

Sciences - Papers: Part A

Sciences

$1-1-2012$

\title{
Experimental research on size effect of micro upsetting of pure copper
}

Guangchun Wang

Shandong University, wang@uow.edu.au

Wei Zheng

Shandong University

Hua Jiang

Shandong University

Zhengyi Jiang

University of Wollongong, jiang@uow.edu.au

Follow this and additional works at: https://ro.uow.edu.au/eispapers

Research Online is the open access institutional repository for the University of Wollongong. For further information contact the UOW Library: research-pubs@uow.edu.au 


\section{Experimental research on size effect of micro upsetting of pure copper}

\section{Keywords}

micro, upsetting, experimental, pure, copper, size, research, effect

\section{Publication Details}

Wang, G., Zheng, W., Jiang, H. \& Jiang, Z. (2012). Experimental research on size effect of micro upsetting of pure copper. Jixie Gongcheng Xuebao/Journal of Mechanical Engineering, 48 (14), 32-37. 


\title{
纯铜微镦粗过程尺寸效应的试验研究
}

\author{
. 王广春 ${ }^{1}$ 郑 伟 $^{1}$ 姜 华 $^{1}$..姜正义 ${ }^{2}$.
}

(1. 山东大学液固结构演变与加工教育部重点实验室 济南 250061;

2. 伍伦贡大学机械、材料和机电一体化学院. 伍伦贡 2522 澳大利亚)

摘要: 在微勄性成形试验机上对纯铜四柱试样进行恒定速度的微锁柤试验. 通过热处理和栺细线切割技术, 设计不同的试样 尺寸和品粒尺寸. 分别研究品粒尺寸和试样尺寸对微望性成形的影响。研究发现: 试样流动应力随着试样尺寸的减小而减小, 其减小趋势与衣面品柆体积分数存在线性关系; 当诖样尺寸较大时, 品粒尺寸对试样流动应力的影响并不明显, 而随葆试样 尺寸的减小, 大尺寸品粒试样的流动应力要明显低于小晶粒尺寸的试样; 当试样尺寸较小时, 试样在微锁粗过程中表现出明 显的丕均匀性. 通过表面层理论和细唱强化对有试样尺寸和品柆尺寸变化引起的尺寸效应现像进行解释, 同时, 引入尺度参 数招化由试样尺寸引起的尺寸效应现象, 从而对当应变一定时流动应力随着衣面层品粒体积分数 $\varphi$ 呈现近似线性变化的原因 进行解释。

关键词: 微成形，锁柤 流动应力，尺寸效应

中图分类号: TG316

\section{Experimental Research on Size Effect of Micro Upsetting of Pure Copper}

\author{
WANG Guangchun ${ }^{1} \cdot$ ZHENG Wei $^{1}$ JIANG Hua ${ }^{1}$ JIANG Zhengyi ${ }^{2}$ \\ (1. Key Laboratory for Liquid-solid Structural Evolution and Processing of Materials of \\ Ministry of Education, Shandong University, Jinan 250061; \\ 2. School of Mechanical, Materials and Mechatronic Engineering; \\ University of Wollongong, Wollongong 2522,'Australia)
}

\begin{abstract}
The process of pure copper micro-cylinder upsetting is performed by a micro universal testing machine. Through the heat treatment and precision wire cutting technology, the specimens are designed with different dimensions and grain sizes. The effects of grain size and specimen size on the flow stress are investigated, respectively. It is found that the flow stress decreases with the miniaturization of the specimen size and the relationship between flow stress and volume fraction of surface grain is linear. The effect of grain size on the flow stress is indistinct as the specimen is larger, but the flow stress of the specimen with larger grain size is obviously lower than that with small size. The surface of the micro-forged specimens show significant inhomogeneous deformation as the specimen size is smaller. The size effects due to the changing of specimen size and grain size can be explained by surface model and fine grain strengthening theories. Meanwhile, the scale parameter is introduced to quantify the size effect and thus the linearly changing of follow stress with the volume fraction $\varphi$ can be explained.
\end{abstract}

Key words: Micro-forming Upsetting Flow stress Size effect

\section{0 前言}

随着电子工业及精密机械的飞速发展, 产品微 型化已成为工业界的发展趋势之一。而传统的基于 机械加工、激光等的微细加工技术已经不能满足微

* 回家白然科学基金 (50975163) 和教有部长江学考及创新团队 (IRT0931)资助项目. 20120201 收到初标, 20120601 收到位改构
型零件批量制造的要求。因此, 低成本和高效率的 微塑性成形工艺成为了微细加工领域的重要发展趋 势。微塑性成形技术是指利用材料的塑性变形来生 产至少在两维方向上尺寸处于 $1 \mathrm{~mm}$ 以下零件的技 术 ${ }^{[1-2]}$, 然而微塑性成形主不是传统塑性成形工艺简 单的几何缩小。由于晶粒尺寸和粗糙度等参数并不 随着加工尺寸的减小而变化, 导致微塑性成形工艺 中出现了有别于传统宏观塑性成形的尺寸效应现 
象 $^{[3-4]}$ 。因此, 对尺寸效应的研究成为了指导微塑性 成形工艺的基础。

在微塑性成形过程中, 材料本身的性能对零件 和工艺设计都有非常重要的意义。因此, 与材料性 能有关的尺寸效应的研究成为微塑性成形研究的热 点。ENGEL 等 ${ }^{[S]}$ 在对 CuZn15 微钽粗试验的研究中 发现: 相同昆粒尺寸下, 当试样尺寸减小时, 材料 的流动应力也随之减小。KALS 等 ${ }^{[6]}$ 在对 CuNi18Zn20 薄板拉伸试验的研究中也发现: 当板 料厚度由 $1.0 \mathrm{~mm}$ 减小到 $0.1 \mathrm{~mm}$ 时, 流动应力明显 减小。KRISHNAN 等[7]对品粒尺寸为 $211 \mu \mathrm{m}$ 的 $\mathrm{CuZn} 30$ 试样进行微挤压试验研究时发现: 当挤出 部分直径小于 $1 \mathrm{~mm}$ 时, $60 \%$ 的试样挤出部分出现了 弯曲, 这说明微成形过程中单个晶粒对整个变形的 影响变大了。然而, 对尺寸效应现象及其产生机理 的认识仍然不足。

在微塑性成形工艺中, 微销粗试验是研究微塑 性成形过程中材料尺寸效应的主要手段。本文设计 了不同的试样尺寸和晶粒尺寸, 对微塑性成形过程 中的尺寸效应进行了研究。

\section{1 纯铜微镦粗试验设计}

\section{1 试样的制备与预处理}

微锁粗的试验材料选用的是电子行业广泛使用 的纯铜。为了得到尺寸微小的圆柱销粗件, 试验选 取直径不同的纯铜铜丝作为原始坯料。在惰性气体 保护状态下对坏料分别采用 $700{ }^{\circ} \mathrm{C}$ 加热 $8 \mathrm{~h}$ 和 700 ${ }^{\circ} \mathrm{C}$ 加热 $24 \mathrm{~h}$ 两种退火热处理工艺, 以便获得不同 的晶粒尺寸 ${ }^{[8]}$ 。图 1 为不同热处理工艺所得到的晶 粒尺寸。通过测焦, 两种热处理条件下得到的平均 昆粒尺寸分别为 $35 \mu \mathrm{m}$ 和 $46 \mu \mathrm{m}$ 。 (a) 700 C加热 $8 \mathrm{~h}$

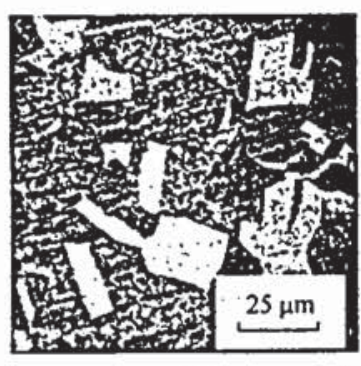

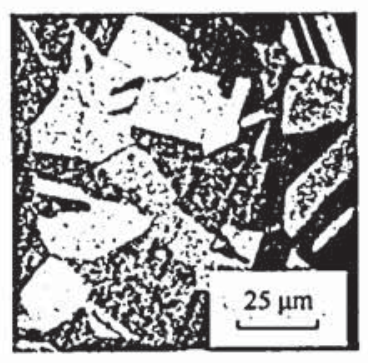

(b) $700 \mathrm{C}$ 如热 $24 \mathrm{~h}$
图 1 两种不同热处理状态下的试件唱粒金相照片

由于坏料尺寸非常微小, 传统的机加工工艺很 难对坯料进行夹持, 坯料表面对划痕、毛刺等缺陷 也很敏感，因此坏料采用精细电火花线切割加工。 为了保证试验的精挽性, 需要注意以下两个方面: (1) 要保证坯料端面的平行度和端面与轴线的垂直
度; (2) 控制电火花加工的放电些和线切割速度, 以 保证坯料能够及时散热。

将不同直径的铜丝统一加工成高径比为 $1.5: 1$ 的圆柱铚粗试样。试样的直径分别为 $0.6 \mathrm{~mm}$ 、 $0.8 \mathrm{~mm} 、 1.0 \mathrm{~mm} 、 1.5 \mathrm{~mm} 、 2.0 \mathrm{~mm}$ 和 $3.0 \mathrm{~mm}$, 加 工后的试样如图 2 所示。

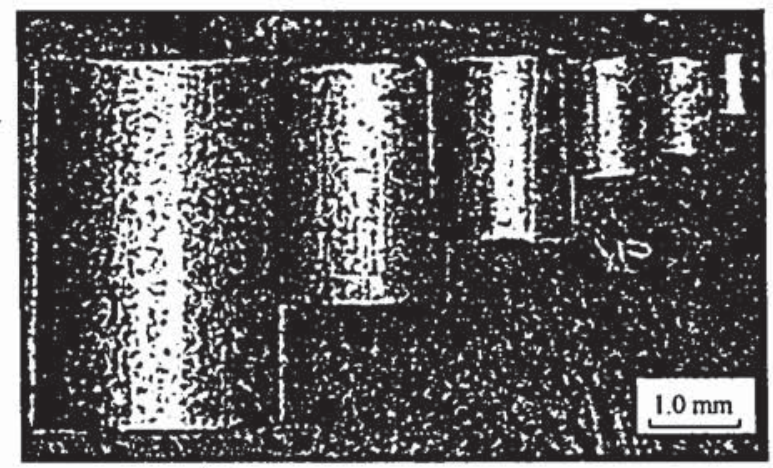

图 2 讯验中采用的不同尺寸的试样

固体润滑剂颗粒若分布不均会对试验结果会产 生很大的影响。而采用液体洞滑剂时, 由于试样与 工具表面之间存在润滑油膜, 易造成试样上下端面 形成自由表面,在降低摩擦对镟粗成形影响的同时, 不受约束的自由表面昆粒沿着最易变形的方向发生 塑性变形而造成衣面凹凸不平 ${ }^{[9]}$ 。为尽可能减小润 滑不均匀对变形的影响, 试验中不采用任何润滑剂。

\section{2 试验条件}

为了尽可能减小摩擦对试验的影响, 皦粗用的 上下压头表面被精加工成镜面, 试样端面的粗糙度 处理成 $0.1 \mu \mathrm{m}$ 以下。试验在室温下进行, 压下速度 为 $0.1 \mathrm{~mm} / \mathrm{s}$ 。微钲粗试验使用位移粮度为 $0.001 \mathrm{~mm}$ 和载荷精度为 $1 \mathrm{~N}$ 的微塑性成形试验机(DT-3AW), 以固定的时间频率实时采集变形过程中的成形力和 位移。试验设备和模具如图 3 所示。

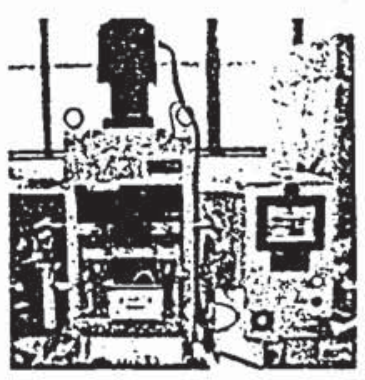

(a) 微成形话较机

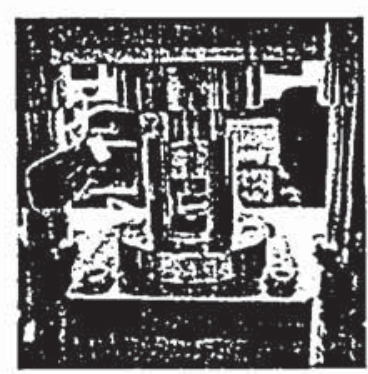

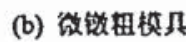

图3试验设各和模具

\section{2 纯铜微镦粗试验结果分析}

\section{1 流动应力}

材料的流动应力是衡量材料塑性性能的主要标 准。由于可以真实地反映瞬时的变形程度和变形抗 
力之间的关系，正应力应变曲线具有更普遍的意义。 根据式(1)处理采集的微钽粗数据可以得到材料的 正应力应变关系曲线 ${ }^{[10]}$

$$
\left\{\begin{array}{l}
\varepsilon=\ln \frac{H_{0}}{H} \\
\sigma=\frac{F}{A_{0} \exp \varepsilon}
\end{array}\right.
$$

式中, $H_{0}$ 和 $A_{0}$ 分别为坯料的初始高度和横截面积, 坏料实时高度 $H$ 和成形力 $F$ 可以通过试验设备进行 采集。微塑性成形中材料的力学性能受两种尺度的 影响: 一种是晶粒尺寸对微塑性成形的影响; 一种 是由产品或坯料空间尺寸微小化对微塑性成形的 影响。

\subsection{1 试样尺寸的影响}

为了研究试样尺寸对流动应力的影响, 对直径 分别为 $0.6 \mathrm{~mm} 、 0.8 \mathrm{~mm} 、 1.0 \mathrm{~mm} 、 1.5 \mathrm{~mm} 、 2.0 \mathrm{~mm}$ 和 $3.0 \mathrm{~mm}$, 晶粒尺寸为 $46 \mu \mathrm{m}$ 的微圆柱试样进行㬚 粗试验。通过多次重复试验取平均值的方法求得不 同试样尺寸下的材料正应力应变曲线如图 4 所示。 从图 4 中可以看出：随着样件尺寸的减小, 流动应 力呈现減少的趋势。这种现象可以采用表面层模型 理论来解释 ${ }^{[11]}$ 。表面层模型认为, 在小尺度情况下, 表面层晶粒增多, 表面层变厚。根据金属物理原理, 与材料内部晶粒相比, 表面层晶粒所受约束限制较 小, 在较低的流动应力下就能变形, 这样变形体的 整体应力就会降低, 而且晶粒尺寸不变时, 随着试 样尺寸的减小, 其比裴面积增大, 这种趋势更加明 显 ${ }^{[12]}$ 。

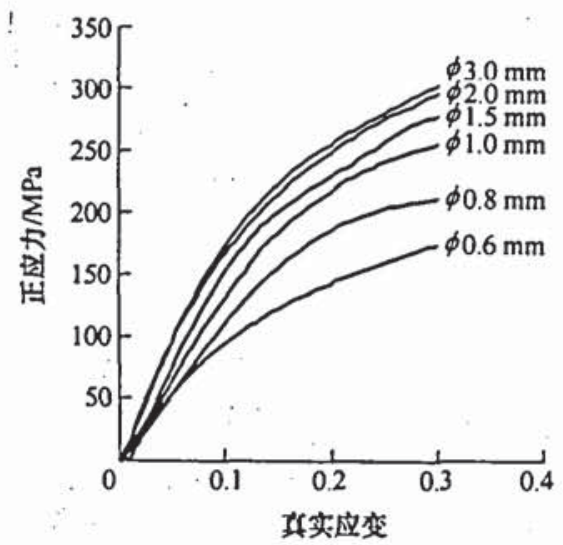

图 4 试样尺寸对流动应力的影响

这里引入尺寸参数 $\varphi$ 来描述装面层晶粒体积分 数。图 5 为圆柱试样的二维截面品粒分布示意图。 其中, 截面上的晶粒由契面层品粒和内部晶粒两部 分组成, 晶粒假设为正六面体, 晶粒的尺寸为 $d$, 试样直径 $D$, 高度 $H$ 。则可知, 表面层晶粒体积 分数

$$
\varphi=1-\frac{(D-2 d)^{2}(H-2 d)}{D^{2} H}
$$

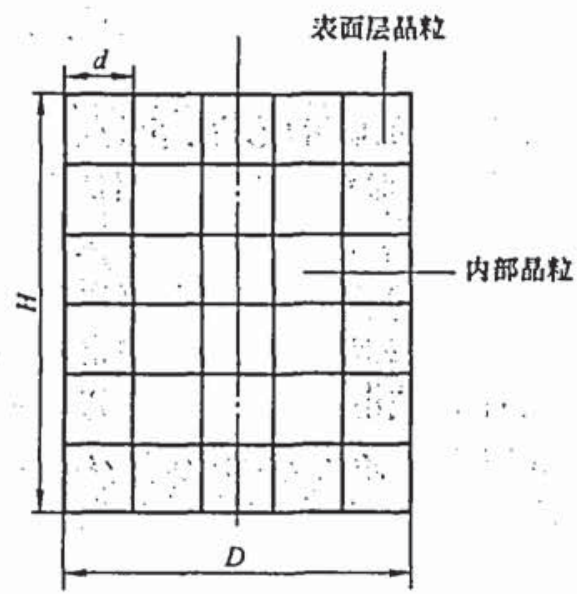

图 5 陶柱截面晶粒分布示意图

根据式(2)可得到高径比 $1.5: 1$, 晶粒尺寸为 46 $\mu \mathrm{m}$ 的圆柱试样的直径与契面层晶粒体积分数 $\varphi$ 的 关系, 如图 6所示。从图 6 中可以看出, 当试样直 径小于 $1.0 \mathrm{~mm}$ 时, 表面层晶粒体积分数 $\varphi$ 已大于 $20 \%$, 这也是试样小于 $1.0 \mathrm{~mm}$ 时, 材料流动应力迅 速减小的原因。

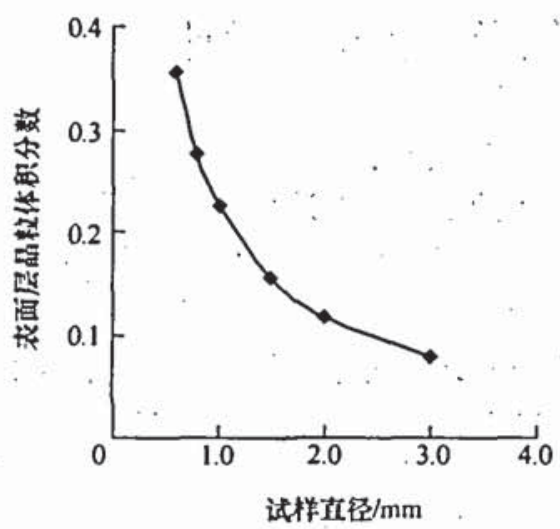

图 6 试样尺寸与表面层晶粒体积分数 $\varphi$ 的关系

通过对不同直径试样流动应力的分析发现: 相 同应变下, 流动应力随着 $\varphi$ 的增加基本呈现线性下 降的趋势, 如图 7 所示。微塑性成形材料的流动应 力可以理解表面层晶粒应力 $\sigma_{\text {surf }}$ 和内部晶粒应力 $\sigma_{\text {in }}$ 共同组成。假设不同表面层晶粒体积分数 $\varphi_{1}$ 和 $\varphi_{2}$ 下的流动应力分别为 $\sigma_{1}$ 和 $\sigma_{2}$, 则有以下关系

$$
\left\{\begin{array}{l}
\sigma_{1}=\varphi_{1} \sigma_{\text {surf }}+\left(1-\varphi_{1}\right) \sigma_{\text {in }} \\
\sigma_{2}=\varphi_{2} \sigma_{\text {surf }}+\left(1-\varphi_{2}\right) \sigma_{\text {in }}
\end{array}\right.
$$

由此可知, 图 7 曲线中两点 $\left(\varphi_{1}, \sigma_{1}\right)$ 和 $\left(\varphi_{2}, \sigma_{2}\right)$ 之间的 斜率

$$
K=\frac{\sigma_{1}-\sigma_{2}}{\varphi_{1}-\varphi_{2}}=\dot{\sigma}_{\text {surf }}-\sigma_{\text {in }}
$$

相同应变下 $\sigma_{\text {surf }}$ 和 $\sigma_{\text {in }}$ 都是定值, 这也是当应变一定， 
时流动应力随着表面层唱粒体积分数 $\varphi$ 呈现近似线 性变化的原因。

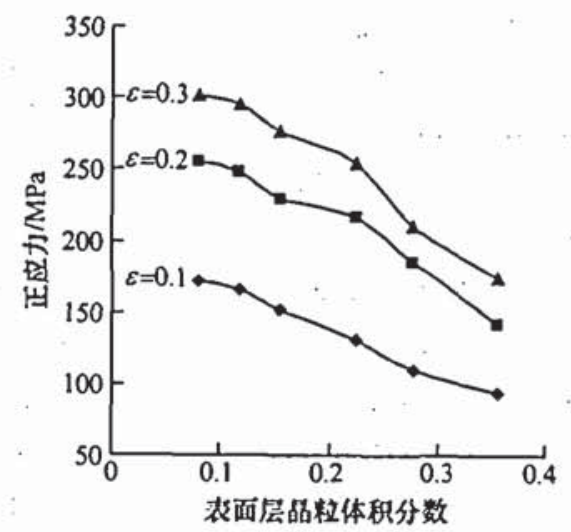

图 7 表面层晶粒体积份数 $\varphi$ 对流动应力的影唃

\subsection{2 晶粒尺寸的影响}

细小的晶粒可以改善多品体材料强度, 这一点 在微观组织对宏观成形的影响中已经被证实了, 并 可以根据位错塞积理论来解释 ${ }^{[13]}$ 。而对于微型件的 成形, 晶粒尺寸的大小还决定着表面层晶粒的体积 分数, 从而进一步影响到材料的流动应力。

图 8 是平均品粒尺寸分别为 $35 \mu \mathrm{m}$ 和 $46 \mu \mathrm{m}$, 试样尺寸分别为 $\phi 3.0 \mathrm{~mm} \times 4.5 \mathrm{~mm}$ 和 $\phi 0.6 \mathrm{~mm} \times 0.9$ $\mathrm{mm}$ 时的销粗压缩时的流动应力一应变曲线。当试 样尺寸为 $\phi 3.0 \mathrm{~mm} \times 4.5 \mathrm{~mm}$ 时, 表面层晶粒的体积分 数很小, 可以认为工件为宏观成形件, 此时由于细 晶强化的作用, 晶粒尺寸为 $35 \mu \mathrm{m}$ 的试样的流动应 力要略大于唱粒尺寸为 $46 \mu \mathrm{m}$ 的试样的流动应力, 如图 8a 所示, 但二者在流动应力上的差别并不明 显, 这主要是由于两种热处理下得到的晶粒尺寸相 差不大导致的。当试样尺寸为 $\phi 0.6 \mathrm{~mm} \times 0.9 \mathrm{~mm}$ 时, 两种晶粒尺寸下的流动应力差别变大, 如图 $8 \mathrm{~b}$ 所 示。这种差别一方面是由于细晶强化的作用造成的, 另一方面是由于晶粒尺寸为 $46 \mu \mathrm{m}$ 的试样其表面层 晶粒体积分数要大于晶粒尺寸为 $35 \mu \mathrm{m}$ 的试样, 而 表面层品粒所受约束限制较小, 这便导致品粒尺寸 为 $46 \mu \mathrm{m}$ 的试样其整体的流动应力下降得更明显。

总之, 就本文的试验结果而言, 几何尺寸对流 动应力的影响较晶粒尺寸的影响要明显。因此, 不 同于宏观成形，在不考虑温度和应变速度的影响的 情况下, 微塑性成形过程中流动应力的主要影响因 素已经不仅仅只有晶粒尺寸, 还包括试样几何尺寸 的影响, 而且几何尺寸越小这种影响越明显。

\section{2 材料不均匀流动}

图 9 为晶粒尺寸 $46 \mu \mathrm{m}$, 试样直径分别为 $0.6 \mathrm{~mm}$

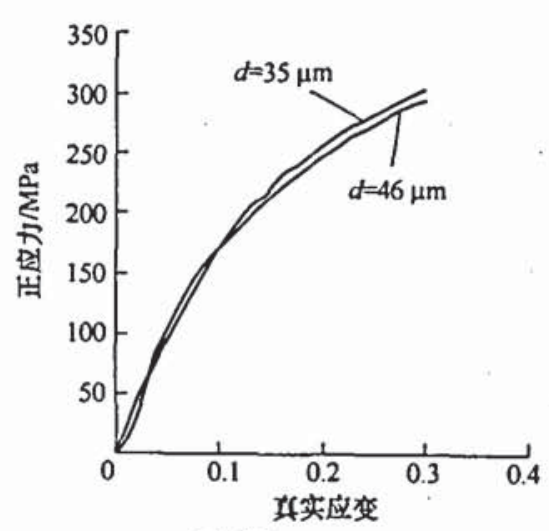

(a) $\phi 3.0 \mathrm{~mm} \times 4.5 \mathrm{~mm}$

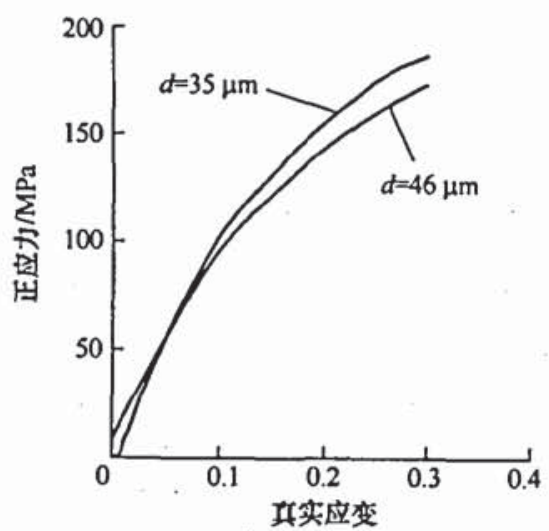

(b) $\phi 0.6 \mathrm{~mm} \times 0.9 \mathrm{~mm}$

图 8 晶粒尺寸对流动应力的影响

和 $3.0 \mathrm{~mm}$ 的试样铰粗后的表面形貌照片。从图 9 中可以看出, 当品粒尺寸一定时, 试样尺寸越小, 变形后的试样表面凹凸不平的程度越大。这说明与 宏观成形不同, 试样在微小尺度情况下, 材料会是 现出不均匀流动的现象。

这种现象可以用金属晶体塑性理论来解释。当 外力作用于多晶体时, 各晶粒滑移系统上的分切应 力因唱粒取向不同而相差很大, 因而各个唱粒的变 形也就不一样。但当试样尺寸处于宏观尺度时, 一 方面由于品粒数陉巨大, 单个晶粒对整个变形的影 响很小, 另一方面由于多晶体中绝大多数晶粒都被 其相邻品粒所包围, 这就要求多品体变形必须依坓 晶粒之间的变形协调来完成, 因此其变形仍表现为 均匀的。

当试样尺寸处于微观尺度时, 试样几何尺寸和 昆粒尺寸之问的比值也随之减小, 这会对微塑性成 形产生如下影响: 一方面构成试样的晶粒总体数目 减少了, 单个㫛粒由于形状和位向的差异而会导致 微塑性成形的不均匀性; 另一方面与自由表面接触 的品粒相对数量却增加了, 这些晶粒本身受到周围 晶粒的约束较小, 这势必加剧材料变形的不均匀性。 


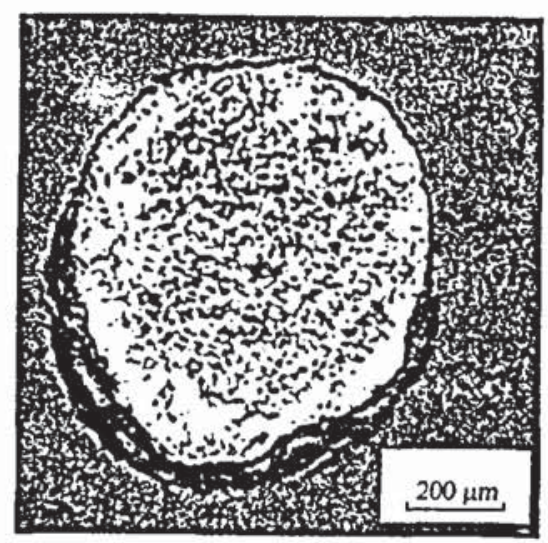

(a) $\phi 0.6 \mathrm{~mm} \times 0.9 \mathrm{~mm}$

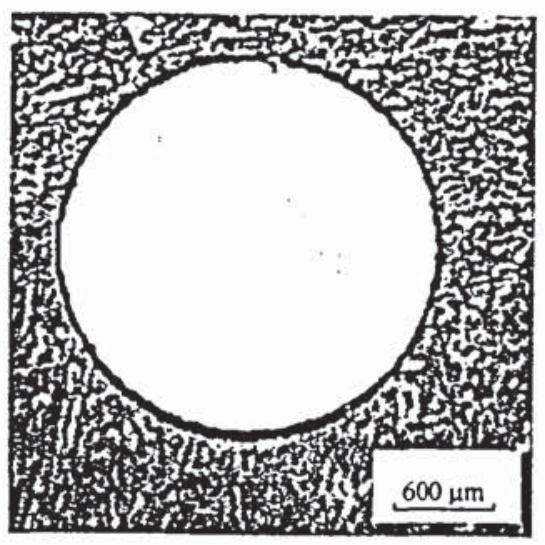

(b) $\phi 2.0 \mathrm{~mm} \times 3.0 \mathrm{~mm}$

图 9 微钽粗后试样的表面形貌

\section{3 结论}

设计不同的试件尺寸和晶粒尺寸对纯铜圆柱微 㬚粗过程进行了试验研究并到得到二者对微塑性成 形材料流动应力的影响规律。

(1) 试样尺寸微小化导致材料流动应力降低及 其变形不均匀性增加, 而在相同试样尺寸下具有较 大晶粒的试样其流动应力要小于具有较小晶粒的试 样, 其原因可以用表面层理论和细品强化理论来 解释。

（2）引入表面层晶粒的体积分数 $\varphi$ 姐化由试样 尺寸引起的尺寸效应现象, 从而对当应变一定时流 动应力随着翌面层晶粒体积分数 $\varphi$ 呈现近似线性变 化的原因进行解释。

\section{参 考 文 献}

[1] 单德枇, 表林, 部坟. 精密微塑性成形技术的现状和发 展趋势[J]. 䘜性工程学报，2008，15(2): 46-53.

SHAN Debin, YUAN Lin, GUO Bin. Research situation and development trends in precision plastic micro forming[J]. Joumal of Plasticity Enginecring; 2008,
15(2): $46-53$

[2] VOLLERTSEN F, HU Z, SCHULZE H, ct al. State of the art in micro forming and investigations into micro deep drawing[J]. Journal of Materials Processing Technology, 2004, 151(1-3): 70-79.

[3] 雷展, 张凯锋. 金属微成形过程中微尺度效应与相似评 估[J]. 机械工程学报, 2007, 43(7): 213-220.

LEI Kun, ZHANG Kaifeng. Micro-size effects and their similarity evaluating methods in metal micro-forming processes[J]. Chinese Journal of Mechanical Engineering, 2007, 43(7): 213-220.

[4] 张凯锋, 王长埘. Zn-Al22 合金超望微成形[J]. 机械工 程学报, 2004, 40(5): 82-86.

ZHANG Kaifeng, WANG Changli. Microforming of superplastic Zn-Al22 alloy[J]. Chinese Journal of Mechanical Engineering, 2004, 40(5): 82-86.

[5] ENGEL U, ECKSTENIN R. Microforming-from basic research to its realization[J]. Journal of Materials Processing Technology, 2002, 125-126(9): 35-44.

[6] KALS TA, ECKSTEIN R. Miniaturization in sheet metal working[J]. Journal of Materials Processing Technology, 2000, 103(1): 95-101.

[7] KRISHNAN N, CAO J, DOHDA K. Study of the size effects on friction conditions in microextrusion-part1: Microextrusion experiments and analysis[J]. Journal of Manufacturing Science and Engineering, 2007, 129(4): 669-676.

[8] 常妍妍. 徽型圆柱件锁相变形规律数值模拟研究[D]. 哈尔滨: 哈尔滨工业大学, 2006.

CHANG Yanyan. Research on numerical simulation of micro copper ; cylinder-upsetting deformation process[D]. Harbin: Harbin Institute of Technology, 2006.

[9] EBRAHIMI R, NAJAFIZADEH A. A new method for evaluation of friction in bulk metal forming[J]. Journal of Materials Processing Technology, 2004, 152(2): 136-143.

[10] 俞汉消, 陈金德. 金属塑性成形原理[M[. 北京：机械 工业出版社, 1999.

YU Hanqing, CHEN Jinde. Principles of metal forming[M]. Beijing: China Machine Press, 1999.

[11] GEIGER M, MESSNER A, ENGEL U. Production of microparts-size effects in bulk metal forming, similarity theory[J]. Production Engineering, 1997, 4(1): 55-58.

[12] 郑伟，王广标; 吴海. 微体积成形品界影响区域材料本 构方程的构建及有限元模推[J]. 机械工程学报, 2011, 47(22): 31-35.

ZHENG Wei, WANG Guangchun, WU. Tao. 
Establishment of constitutive equation of material in region affected by grain boundary and numerical simulation of micro-bulk-forming[J]. Journal of Mechanical Engineering, 2011, 47(22): 31-35.

[13] 杨觉先. 金属㕵性变形物理基础[M]. 北京: 治金工业 出版社, 1987.

YANG Juexian. Physical basis of metal forming[M]. Beijing: Metallurgical Industry Press, 1987.

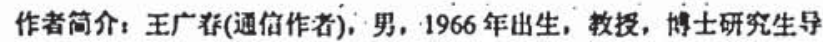
师. 主要研究方向为金風筀性成形数值摸投与优化技术及块速成形与块 速拱具制造技术.

E-mail:wgc@sdu.edu.cn

郑伟, 男, 1982 年出生, 博士研究生. 主要研究方向为微成形数值措报 方法及技术.

E-mail: zhengweinol@163.com

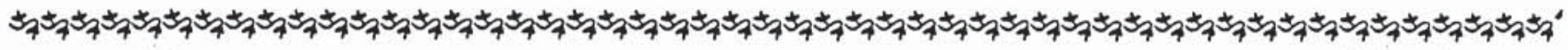

\section{《机械工程学报》.被 EI 收录论文目次(2011 年第 21 期)}

暒于多约束的机器人关节空间轨迹规划 张 斌 (1)

仿壁鶴脚趾结构设计及粘附运动性能测试 俞志伟 李宏凯 张晓峰等 (7)

基于概率分布的 3-UPU 机构误差影响钽感度 邦盛 王乃明 方跃法等 (14)

基于人工免投反浈的自治水下机器人推力器控制 吴乃龙 刘责杰 李思乐等 (22) 多妳带传动系统轮一苗振动的实测与计55方法研究 上官文斌，张，智、许秋海 (28) 聯峦式爬壁机器人的研制 阵胜栍 任启乐 陈正文等 (37)

空问共轮啮合别二次包络面三面共点构造法 李钥上 (43)

带弹性环保护轴承的动力学特性 朱益利 徐龙祥 (49)

采用粒子群算法的冲出信乓自适应单稳态随机坛据检测方法 本继猛 陈雪峰 何正态 (58) 登于有限元法的周期拱形结构振动特性

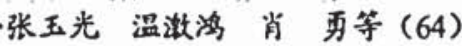
磁悬浮球形关节三维动力学模型与控制特性 曾 励 张 帆 徐媛境 (69) 行星齿轮系扭转非线性振动建模与运动分分特性研究 李同杰 朱如垉和云等 (76) 含裂纹故帟业轮系统的非线性动力学研究 ：锐，陈子恕:(84) 线接触海一流条件下微凹坑㳖面磨擦特性

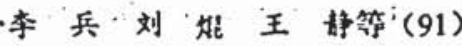
钴势合金的沙动磨损行为及仿真预测 李联联 曾 聚 雷丽萍等 (97) 䄳于 Navier 滑移的油膜继际微流动特性数值分析 刘赵粶，王国斌中，㸼（104） 一种结合部法向刚度的预估方法 王世军 赵金娟 张照军等 (111)

一种支持型腔租加工刀具序列高效优化的刀具的选方法 尹布㱛 刘飞刘霜等 (116) 基于技术永交的一类产品技术集成创新设计

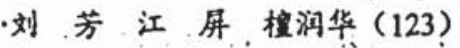
废弃电路板插装件的自适应磨洌拆解方法 龙旦风 向 东 董衵弘等 (133) 一种基于硬件在环的可亚构数控系统验证方法 杜少华 于 东 黄：绝等 (139) 基于扩展干涉矩阵的几何可拆缷性判别方法 于嘉鵖 那宇飞王成思（146） 光制造科学与技术的现状和展望 王国被 (157) 証于锯切弧区切向力分布的功率消枆模型

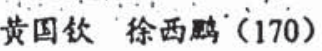

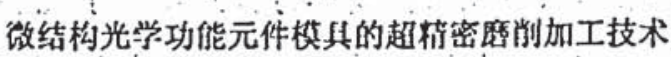
赵清竞 邦 兵 (177)

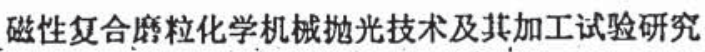

